Revue internationale P.M.E.

Économie et gestion de la petite et moyenne entreprise

\title{
L'évaluation des projets d'innovation de procédés dans les PME manufacturières : difficultés d'application des méthodes \\ financières conventionnelles
}

\author{
Josée St-Pierre et Robert Beaudoin
}

Volume 9, numéro 2, 1996

URI : https://id.erudit.org/iderudit/1008261ar

DOI : https://doi.org/10.7202/1008261ar

Aller au sommaire du numéro

Éditeur(s)

Presses de l’Université du Québec

ISSN

0776-5436 (imprimé)

1918-9699 (numérique)

Découvrir la revue

Citer cet article

St-Pierre, J. \& Beaudoin, R. (1996). L'évaluation des projets d'innovation de procédés dans les PME manufacturières : difficultés d'application des méthodes financières conventionnelles. Revue internationale P.M.E., 9(2), 37-56. https://doi.org/10.7202/1008261ar
Résumé de l'article

Pour évaluer la rentabilité financière d'un projet d'implantation d'un système de gestion juste-à-temps dans les PME manufacturières, nous avons développé une grille résumant l'ensemble des bénéfices et des coûts facilement, difficilement et non quantifiables, susceptibles d'être modifiés par un tel projet. Nous appliquons ensuite le modèle proposé sur des données financières simulées à partir de cas que nous avons analysés. Les résultats obtenus montrent l'importance de procéder à une analyse du type proposé pour obtenir une évaluation minimale du rendement espéré et surtout, du risque que représente un tel projet d'innovation. Contrairement à ce qu'affirment plusieurs auteurs, nos résultats indiquent que les PME ne peuvent se soustraire à l'évaluation financière de ces projets, étant donné les différents éléments de risque qu'ils comportent et l'incertitude de leur réussite. 


\title{
L'évaluation des projets d'innovation de procédés dans les PME manufacturières: difficultés d'application des méthodes financières conventionnelles
}

\author{
Josée ST-PIERRE \\ Robert BEAUDOIN \\ Université du Québec à Trois-Rivières
}

\section{MOTS CLÉS}

\section{Évaluation financière - Évaluation de projet - Prise en compte du risque - Juste-à-temps - Projet d'innovation}

\section{RÉSUMÉ}

Pour évaluer la rentabilité financière d'un projet d'implantation d'un système de gestion juste-à-temps dans les PME manufacturières, nous avons développé une grille résumant l'ensemble des bénéfices et des coûts facilement, difficilement et non quantifiables, susceptibles d'être modifiés par un tel projet. Nous appliquons ensuite le modèle proposé sur des données financières simulées à partir de cas que nous avons analysés. Les résultats obtenus

\section{LES AUTEURS}

Josée St-Pierre est titulaire d'un doctorat en finance (Ph.D.) de l'Université Laval. Elle est l'auteure de plusieurs articles scientifiques dans les domaines de la régie d'entreprises, des offres publiques d'achat et du financement par projet. Ses intérêts de recherche portent également sur l'étude des problèmes financiers des PME. Elle est membre du GREPME depuis 1993.

Robert Beaudoin est détenteur d'un doctorat en gestion de l'Université Aix-Marseille III. Ses intérêts de recherche portent principalement sur l'étude des problèmes financiers des PME. II a œuvré pendant plusieurs années comme cadre supérieur à l'Université du Québec à Trois-Rivières. II est membre du GREPME depuis 1994.

Adresse: Université du Québec à Trois-Rivières, 3351, boul. des Forges, C.P. 500, Trois-Rivières (Québec), G9A 5H7. 
montrent l'importance de procéder à une analyse du type proposé pour obtenir une évaluation minimale du rendement espéré et surtout, du risque que représente un tel projet d'innovation. Contrairement à ce qu'affirment plusieurs auteurs, nos résultats indiquent que les PME ne peuvent se soustraire à l'évaluation financière de ces projets, étant donné les différents éléments de risque qu'ils comportent et l'incertitude de leur réussite.

\begin{abstract}
In this paper, we present a model for evaluating a technology investment: the implantation of a just-in-ime production system in manufacturing SME. To ascertain the risk of the project, we introduce quantifiable and non quantifiable elements of benefits and costs, in the evaluation. We interviewed entrepreneurs of SME, analyse their financial statements and elaborate a case, to test our model. In doing so, we demonstrate the importance of evaluating this kind of project, even if classical financial tools are not adapted to their specificities. Contrary to what many authors said, SME can't adopt a new production system as just-in-time, without a financial evaluation. The project is too risky and the incertainty is too high.
\end{abstract}

\title{
RESUMEN
}

Para evaluar la rentabilidad financiera de un proyecto de implantación de un sistema de gestión "justo a tiempo ", en las PyME de manufactura, hemos desarrollado una guía que resume el conjunto de beneficios y de costos financieros determinables o no y a la vez suceptibles des ser modificados por un tal proyecto. Luego aplicamos el modelo propuesto sobre datos simulados a partir de casos que hemos analizado. Los resultados obtenidos muestran la importancia de proceder a un análisis del tipo propuesto, para obtener una evaluación minima del rendimineto esperado y sobretodo del riesgo que representa un tal proyecto de innovación. Contrariamente a lo que afirman varios autores, nuestros resultados indican que las PyMEs no pueden abstenerse de proceder a la evaluación financiera de estos proyectos, dado el caso de los diferentes elementos de riesgo que los rodean y la incertidumbre en cuanto al exito del proyecto.

\section{Introduction}

Le concept de production juste-à-temps (ci-après JAT) a été développé dans l'économie japonaise, où un certain nombre de facteurs ont contribué à son succès. On le définit comme :

[...] une approche de la gestion de la production qui vise à améliorer la productivité et la qualité des systèmes manufacturiers afin de les rendre plus compétitifs. Cette approche repose sur trois concepts de base : intégration et optimisation, amélioration constante du système, relations avec les clients et les fournisseurs. (Ramudhin et Marier, 1992) 
L'objectif d'un système de gestion JAT est de débarrasser le processus de production de toutes ses sources d'inefficacité ou de gaspillage en resserrant les différents contrôles, en réduisant les temps de mise en course, en améliorant le contrôle de la qualité et, de façon plus générale, en éliminant tous les efforts non nécessaires dans le processus de transformation des matières premières en produits finis (Jewkes et Power, 1993).

Les principaux facteurs responsables du succès du JAT au Japon sont les suivants : un temps de mise en course rapide des équipements; une maind'œuvre hautement compétente et disciplinée ; un très fort degré d'automatisation du système de production ; un nombre relativement petit de pièces à fabriquer (Philipoom et al., 1987). Un élément important du succès, mais qui n'est pas de nature "opérationnelle », est la volonté de coopération très étroite entre les fournisseurs, les manufacturiers, les employés et les clients qui permet de minimiser la variabilité dans les horaires de production. Cette variabilité est attribuable à la qualité des prévisions de production qui devient dès lors un facteur de succès déterminant.

Par ailleurs, Philipoom et al. (1987) doutent que les succès japonais du JAT puissent être obtenus aux États-Unis, étant donné les différences culturelles et économiques entre les deux pays. Mentionnons, à titre d'exemple, la très grande discipline des employés japonais, ainsi que leur flexibilité, et les relations d'affaires entre clients et fournisseurs qui sont fondées sur le respect. La syndicalisation des entreprises américaines, le fait qu'elles fabriquent plusieurs pièces sur une même chaîne de production, qu'elles fassent en même temps de la production et de l'assemblage, ainsi que la nature des relations entre clients et fournisseurs, sont tous des facteurs qui peuvent contribuer au fait que le succès américain du JAT soit loin d'être assuré. Ces raisons justifient une étude de rentabilité financière détaillée ainsi que des facteurs de risque pouvant compromettre le succès de l'implantation du projet.

L'implantation d'un système de gestion JAT consiste en un projet qui, d'un point de vue financier, doit faire l'objet d'une étude de faisabilité comme tout autre projet d'investissement. Toutefois, la littérature financière n'offre actuellement aucun outil d'analyse nous permettant de procéder de façon systématique à l'évaluation d'un tel projet. La difficulté d'une telle évaluation réside surtout dans la complexité à définir et à mesurer de façon exhaustive l'ensemble des flux monétaires inhérents à ce nouveau mode de gestion (voir entre autres Inman et Mehra, 1993 ; Jewkes et Power, 1993 ; Mackey, 1989; Miltenburg, 1993 ; Primrose, 1992 ; Shank et Govindarajan, 1992). Par ailleurs, le succès obtenu au Japon par cette forme de gestion amène certains dirigeants à juger inutile l'évaluation financière, puisque ces projets sont considérés comme stratégiques et doivent, peu importe le résultat de l'évaluation, être entrepris. On retrouve donc, dans la littérature en gestion de la production, des 
arguments de deux types pour expliquer l'absence de modèles d'évaluation appropriés :

1. Certains investissements considérés comme stratégiques devraient être acceptés sans aucune justification économique ni financière. On argumente alors que ces investissements doivent être réalisés pour maintenir la compétitivité de l'entreprise, car autrement elle risquerait de voir sa part de marché diminuer au profit de ses concurrents qui l'auront devancée sur le plan technologique. Shank et Govindarajan (1992) rapportent que $40 \%$ des entreprises n'utilisent aucune méthode d'évaluation pour justifier la réalisation de projets d'investissement en innovation technologique.

2. Les modèles traditionnels de choix d'investissement ne sont pas adaptés à ce type de projet à cause, en grande partie, de l'incertitude qui n'est pas facilement mesurable, voire pas du tout. On prétend en effet que les méthodes basées sur l'actualisation des flux monétaires conduisent les décideurs à préférer des investissements moins prometteurs à des investissements stratégiques dans des innovations, puisque les bénéfices reliés à ceux-ci sont très incertains et difficilement quantifiables. Plutôt que d'utiliser une méthode d'évaluation mal adaptée pour mesurer la rentabilité d'un projet, on préfère justifier sa réalisation par le fait que les autres le font! (Primrose, 1992)

Dans cette recherche, nous allons étendre le concept de flux monétaires traditionnellement utilisés en finance et présenter une grille d'analyse qui tient compte à la fois des bénéfices et des coûts facilement et difficilement quantifiables imputables à un projet d'implantation de JAT. Cette grille nous permettra par la suite de simuler un traitement du risque dans l'évaluation de la rentabilité financière de ce type de projet.

Pour élaborer notre grille et notre modèle d'analyse, nous avons utilisé l'implantation du JAT dans les PME manufacturières. Nous avons eu accès aux diverses données financières et avons procédé à plusieurs entrevues avec les dirigeants d'un groupe de PME et les responsables des implantations, afin de pouvoir déterminer les coûts et bénéfices impliqués. Les observations recueillies ont ensuite servi à rédiger un cas permettant d'illustrer la façon d'analyser financièrement un projet d'investissement dans les innovations de procédé.

Après avoir rappelé les principes de l'évaluation financière d'un projet, nous expliquerons les particularités du JAT et leurs effets sur l'évaluation financière ainsi que les différents éléments de risque. Nous proposerons ensuite un modèle d'analyse du JAT plus complet, dont l'utilisation sera illustrée par une simulation à partir d'une étude de cas. Nous formulerons finalement nos conclusions. 


\section{L'évaluation de projets d'innovation : cadre d'analyse traditionnel et particularités du JAT}

\subsection{Le modèle d'évaluation classique pour déterminer la rentabilité}

On sait que parmi les différentes méthodes d'évaluation de projets qu'offre la théorie financière, ceux qui prennent en compte l'aspect temporel des flux monétaires en faisant appel au principe de l'actualisation sont significativement supérieurs aux autres ${ }^{1}$. Or, pour utiliser correctement ces modèles, l'analyste doit disposer du spectre le plus large possible des effets du projet sur l'ensemble de l'entreprise.

Rappelons la formule de calcul de la valeur actuelle nette:

$\sum_{t=0}^{\mathrm{N}}\left[\frac{(\text { Flux monétaires nets })_{t}}{(1+k)^{t}}\right]+\sum_{t=0}^{\mathrm{N}}\left[\frac{(\text { Conséquences fiscales des investissements })_{t}}{(1+k)^{t}}\right]-\sum_{t=0}^{\mathrm{N}}\left[\frac{\text { (Investissements) } t}{(1+k)^{t}}\right]$

où $k$ exprime le taux d'actualisation ajusté au risque du projet évalué; $t$ indique la période de réalisation du flux monétaire ; et $N$ représente la durée du projet. La détermination de chacun des éléments de la formule présente certaines particularités et complexités selon la nature du projet évalué. Par exemple, la durée du projet peut être difficile à déterminer dans le cas du lancement d'un nouveau produit où l'on ignore la situation des concurrents, soit leur degré d'avancement sur un projet similaire. Nous traiterons plus loin des difficultés à déterminer la valeur du paramètre $k$ pour l'évaluation de projet d'innovation et de la prise en compte du risque.

Dans le cas des flux monétaires futurs liés au projet, plusieurs méthodes peuvent permettre de fournir des estimations relativement pertinentes ${ }^{2}$. On peut utiliser les données historiques de l'entreprise, dans le cas de projets routiniers, que l'on ajuste pour tenir compte de l'évolution de la situation par rapport au

1. Malgré les avantages que présentent les modèles d'actualisation, notamment celui de la valeur actuelle nette (VAN), Jog et Srivastava (1994) ont montré qu'encore peu d'entreprises canadiennes y faisaient régulièrement appel pour évaluer leurs projets. Les auteurs n'ont toutefois pas nuancé leur étude comme ils auraient pu le faire en soulevant la question de la cohérence entre l'importance du projet étudié et le choix du modèle d'évaluation.

2. Behrens et Hawranek (1993) présentent l'ensemble de la démarche servant à l'évaluation des différents projets industriels, allant des études de préfaisabilité jusqu'à la décision finale d'acceptation ou de rejet du projet. Pour un relevé de la littérature, voir Gélinas et al., 1994. 
dernier projet similaire évalué. S'il s'agit d'un nouveau projet et que l'entreprise ne dispose d'aucune expérience pour évaluer les flux, on fera appel à différents intervenants dans l'entreprise ou à des consultants. Pour lancer un nouveau produit, par exemple, les études de marché, environnementales, sociales, d'ingénierie, etc. serviront à rassembler les informations que l'analyste financier pourra transformer en flux monétaires pour l'évaluation. Évidemment, plus le projet présente un caractère innovateur, moins l'analyste dispose d'informations précises et plus il sera complexe d'effectuer une évaluation financière. Dans ces conditions, différentes approches de simulation ou de projections de scénarios pourront améliorer la qualité du résultat de l'analyse financière et fourniront de meilleurs outils pour la prise de décision.

Ces propos nous amènent à rappeler que la qualité de la décision prise au regard d'un projet d'investissement dépend de l'information fournie par l'évaluation financière qui, elle, dépend de la qualité des données qui ont servi à produire le résultat final. Aucune décision rationnelle ne pourra être prise si l'évaluation financière n'a pas été réalisée soigneusement en tenant compte de toutes les difficultés qu'elle implique. Nous avons déjà souligné les problèmes d'estimation des flux monétaires dans le cas de projet routinier, et nous allons maintenant démontrer que les projets d'innovation présentent une complexité encore plus grande.

\subsection{Les effets potentiels du JAT et l'évaluation financière}

Plusieurs articles publiés dans des périodiques en gestion de la production montrent les avantages du JAT en faisant ressortir surtout les réductions de coûts possibles dans le processus de production ${ }^{3}$. On y indique alors que les profits de l'entreprise augmenteront étant donné les effets occasionnés par:

- une réduction des temps de mise en course et des délais de fabrication;

- une augmentation de la qualité et, par conséquent, une réduction du nombre de pièces rejetées ;

- une réduction du niveau des inventaires et des frais afférents;

- une augmentation de la productivité de la main-d'œuvre ;

- une augmentation de la flexibilité ;

- une réduction de la surface de plancher nécessaire à la production ;

- une réduction des frais généraux de fabrication (Primrose, 1992).

3. Pour un relevé de la littérature, voir Gélinas et al., 1994. 
Par ailleurs, l'augmentation de la flexibilité de la production et de la qualité des produits finis aura des conséquences sur le chiffre d'affaires de l'entreprise et sur son niveau de ventes dans le futur (Jewkes et Power, 1993). Comme ces éléments sont difficilement quantifiables et reposent en bonne partie sur une évaluation subjective des décideurs, on les omet souvent dans l'analyse de l'évaluation de la décision d'adopter ou non le JAT.

Finalement, si les bénéfices du JAT proviennent de l'amélioration des délais de livraison qui amène une augmentation des ventes, les dirigeants devront soit augmenter leurs stocks de produits finis, soit accroître la cadence de production (Primrose, 1992). Le décideur pourra alors être amené à faire un choix entre produire de façon irrégulière et ne maintenir aucun inventaire, ou lisser sa production, subir des frais de stockage et accepter le risque de désuétude des unités fabriquées. On voit donc, dans un tel contexte, que la réduction des inventaires n'est pas nécessairement un bénéfice occasionné automatiquement par le JAT.

À cet égard, Billesbach et Hayen (1994) ont étudié les données relatives aux inventaires de 28 grandes sociétés américaines ayant affirmé avoir adhéré au mode de gestion JAT. Bien qu'ils observent une plus grande rotation des stocks entre les périodes pré- et post-JAT, les auteurs ne peuvent conclure à une amélioration de l'efficience étant donné l'absence de mesures de performance relative. Par ailleurs, Inman et Mehra (1993) ont interrogé des entreprises manufacturières américaines (PME et GE) qui ont affirmé avoir amélioré la rotation de leurs inventaires de $52 \%$ en moyenne, après l'adoption du JAT. Aucune mesure de performance relative n'a toutefois été utilisée dans leur étude afin de valider ce résultat.

Primrose (1992) affirme que les entreprises qui considèrent l'adoption de la philosophie JAT ne doivent pas s'attendre qu'à en tirer des bénéfices ; cela peut parfois occasionner des dépenses substantielles. Ce qui implique, selon lui, que l'on doive faire une évaluation financière peu importe l'ampleur des investissements à réaliser. Il soutient de plus que l'absence d'évaluation formelle du JAT peut avoir un certain nombre de conséquences telles que les suivantes :

- 1'attribution de ressources à des activités très peu productives au détriment d'autres activités plus rentables ;

- l'absence d'objectifs quantifiables empêchant les dirigeants de suivre les progrès de l'implantation du JAT et d'en mesurer l'ampleur ;

- le danger d'abandonner l'implantation si la mode change ;

- le fait de retarder des projets prioritaires pour l'adoption du JAT, sans même avoir une idée de la valeur de cette décision. 
Jewkes et Power (1993) concluent leur étude en affirmant que, contrairement à une certaine croyance, l'évaluation de la rentabilité financière ou économique d'un système de gestion JAT doit se faire en adoptant une approche à long terme, étant donné que plusieurs des bénéfices (surtout ceux qui sont difficilement quantifiables) ne se feront pas sentir avant quelque temps.

Les auteurs qui argumentent que le JAT n'a pas besoin d'être l'objet d'une évaluation financière affirment, entre autres, que le dernier terme de l'équation que nous avons présentée plus haut est souvent nul, c'est-à-dire qu'aucun investissement n'est requis pour implanter le JAT qui n'est alors considéré que comme un mode de gestion, ce qui, du même coup, annule le deuxième terme de l'équation. On ne conserve alors que le premier terme qui ne présente que des avantages tels que ceux mentionnés plus haut. On comprendra alors la nonpertinence de l'évaluation financière puisque ce terme ne peut que produire une valeur positive et contribuer à l'enrichissement des propriétaires de la firme.

En résumé, on retrouve plusieurs auteurs qui soutiennent que les projets de production à valeur ajoutée ne nécessitent que peu d'investissements (annulant ainsi les deux derniers termes de l'équation), ne présentent que des avantages (procurant ainsi automatiquement une valeur positive au premier terme) et présentent plusieurs effets difficilement quantifiables qui expriment le caractère stratégique du projet et qui, par conséquent, ne nécessitent aucune justification financière.

Les résultats de nos consultations avec quelques consultants moins dogmatiques et plusieurs dirigeants d'entreprise en processus d'implantation du système, ainsi que le faible taux de succès de l'implantation du JAT nous amènent plutôt à considérer comme très risqué ce genre de projet où le degré d'incertitude des effets oblige à une évaluation financière. Avant de présenter notre modèle, nous allons traiter du risque que présente un projet d'innovation de procédé.

\subsection{Le JAT et les éléments de risque ${ }^{4}$}

On sait que les principaux facteurs de risque compromettant le succès de la plupart des projets d'investissement sont liés à l'augmentation des délais de

4. Les notions de risque et d'incertitude sont évidemment très voisines l'une de l'autre. Behrens et Hawranek (1993, p. 264) précisent que: «[...] il y a risque quand la probabilité de survenance d'un événement peut être exprimée sur la base de données, et incertitude quand cette probabilité doit être définie de manière subjective parce qu'il n'existe pas de données rétrospectives ». Le risque d'un projet $s$ 'accroît avec l'incertitude puisque l'évaluateur ou le décideur n'a pas d'éléments précis pour l'éclairer dans sa prise de décision. 
mise en route du projet : délais de développement d'un nouveau produit, délais de construction, délais de mise en exploitation, etc. L'augmentation des délais retarde la période où devraient commencer à se matérialiser les bénéfices attendus de telle sorte que le promoteur du projet se retrouve avec un important problème de liquidités qu'il ne peut résoudre efficacement que s'il possède une très bonne situation financière.

Dans le cas d'un projet d'innovation de procédé tel que celui sous étude, les facteurs de risque sont principalement liés à l'absence de prise en compte de certains bénéfices et coûts difficilement quantifiables et des effets du projet sur l'ensemble de l'entreprise : capacité des employés à réagir rapidement à des modifications du carnet de commande, grève chez un fournisseur qui interrompt la ligne de production (cas de GM au Michigan, octobre 1994), modification non prévue dans le flux des approvisionnements, dépendance de l'entreprise fournisseur à l'égard de son donneur d'ordre, imprécision des commandes du donneur d'ordre, climat de travail et relations avec le syndicat, etc.

Les méthodes d'évaluation qui consistent à actualiser les flux monétaires (valeur actuelle nette) considèrent le risque, entre autres, dans un taux d'actualisation ajusté (en ajoutant une prime au facteur $k$ de notre équation) et, de ce fait, ne favorisent pas les projets dans les nouvelles technologies où une part importante des bénéfices futurs est qualitative, hautement incertaine et difficilement quantifiable. Certains auteurs (voir Accola, 1994) critiquent le fait qu'en ajustant le taux d'actualisation selon le degré de risque perçu du projet, et en omettant tous les bénéfices secondaires, l'évaluateur considère deux fois le risque dans son projet et, par conséquent, en sous-évalue grandement la rentabilité.

Les critiques d'Accola visent le fait que le numérateur du premier terme du calcul étant sous-évalué par l'absence de prise en compte des éléments difficilement quantifiables, et le dénominateur étant déjà ajusté pour le risque du projet analysé, le risque est donc considéré deux fois. Nous complétons les commentaires d'Accola en affirmant qu'un projet d'innovation de procédé, tel que le JAT, ne représente pas un risque supplémentaire qui s'ajoute au risque global de l'entreprise et qui justifierait un ajustement au taux d'actualisation. Il représente plutôt un haut degré d'incertitude et, de ce fait, devrait simplement être analysé différemment (nous y reviendrons).

Évidemment, le manque d'outil d'analyse complet augmente les possibilités de dérapage de tels projets; puisque s'ils ne sont pas évalués correctement, les différents facteurs de risque ne pourront être contrôlés. 


\section{Une nouvelle approche pour l'évaluation des projets d'innovation de procédé dans les PME}

\section{1. Élaboration d'un modèle d'analyse}

Après avoir interrogé plusieurs dirigeants de PME manufacturières et consulté un certain nombre d'ouvrages en finance, en comptabilité, en production et en ingénierie, nous avons pu élaborer une grille d'évaluation sommaire ${ }^{5}$ (voir le tableau 1) de l'ensemble des effets attendus de l'implantation du juste-à-temps. Dans le tableau 1, nous avons classé les avantages facilement quantifiables comme étant de niveau primaire, les difficilement quantifiables de niveau secondaire et les non-quantifiables de nature plutôt qualitative. Les six catégories de bénéfices énoncés sont ceux que l'on retrouve le plus souvent dans la littérature (Wantuck, 1989; CMA, 1993).

À tous ces bénéfices sont associés un certain nombre de coûts que beaucoup d'auteurs négligent dans leur évaluation des avantages réels du JAT. Mentionnons, à titre d'exemple, que la polyvalence des équipements ainsi que le fait que l'on augmente souvent la cadence de production a pour conséquence d'augmenter la vitesse d'usure de la machinerie. En contrepartie, les dirigeants devraient allouer des sommes supplémentaires pour l'entretien préventif, ce qui permettrait d'augmenter la durée des équipements. Par ailleurs, la polyvalence des employés peut également entraîner des coûts supplémentaires, notamment dans un environnement syndiqué où l'on demande désormais à chaque employé d'accomplir plusieurs tâches à la fois. Nous avons donc repris ces coûts dans un format semblable à celui des bénéfices. De même que pour les bénéfices, nous éprouvons également des difficultés dans l'identification de la totalité des coûts imputés au JAT.

La consultation de la documentation mentionnée plus haut (voir le tableau 2) a permis de décrire relativement facilement les bénéfices et les coûts primaires, plutôt difficilement les éléments secondaires, alors que tout ce qui est de nature qualitative, et qui correspond parfois aux éléments de risque, a pu être développé grâce aux rencontres que nous avons eues avec des dirigeants de PME ayant adopté ou étant en voie d'adopter le JAT. Ces informations sont présentées au tableau 1 . Notez que la présence de cellules vides ne signifie pas qu'il n'existe aucun effet du JAT sur cet élément de coût ou de bénéfice, mais simplement que nous n'avons pas pu en identifier.

Notre démarche se différencie de celle des autres auteurs par l'ajout des éléments qualitatifs ainsi que par l'utilisation d'une approche quantitative

5. On trouvera des grilles plus détaillées dans Pinsonnault (1996) et dans St-Pierre et Beaudoin (1995). 
TABLEAU 1 Grille d'évaluation des bénéfices et des coûts attendus de l'implantation d'un système de gestion juste-à-temps

\begin{tabular}{|c|c|c|c|c|c|}
\hline Éléments primaires & & Éléments secondaires & & Éléments qualitatifs & \\
\hline Bénéfices & Coûts & Bénéfices & Coûts & Bénéfices & Coûts \\
\hline $\begin{array}{l}\text { 1. Cô̂t de revient } \\
\text { Réduction du niveau } \\
\text { minimal d'inventaires. } \\
\text { Réduction du coût des } \\
\text { approvisionnements. }\end{array}$ & $\begin{array}{l}\text { Augmentation du coût des } \\
\text { matières premières. }\end{array}$ & $\begin{array}{l}\text { Réduction des activités } \\
\text { non créatrices de valeur } \\
\text { (fabrication ou acquisition } \\
\text { en quantité non optimale). }\end{array}$ & $\begin{array}{l}\text { Coûts de la réorganisation } \\
\text { (délai d'apprentissage...). }\end{array}$ & $\begin{array}{l}\text { Réduction des coûts } \\
\text { de gestion des imprévus. }\end{array}$ & $\begin{array}{l}\text { Arrêt non prévu } \\
\text { dans le flux des } \\
\text { approvisionnements. }\end{array}$ \\
\hline $\begin{array}{l}\text { 2. Qualité } \\
\text { Réduction des coûts } \\
\text { de défaillance interne. }\end{array}$ & $\begin{array}{l}\text { Augmentation des coûts } \\
\text { en entretien préventif. }\end{array}$ & $\begin{array}{l}\text { Augmentation des ventes } \\
\text { grâce à une amélioration } \\
\text { du rapport qualité / prix. }\end{array}$ & $\begin{array}{l}\text { Augmentation des coûts } \\
\text { du système de garantie } \\
\text { des pièces fabriquées. }\end{array}$ & $\begin{array}{l}\text { Amélioration } \\
\text { de la qualité totale. } \\
\text { Diminution de l'écart } \\
\text { de production vs planifiée. }\end{array}$ & $\begin{array}{l}\text { Dépendance de la } \\
\text { qualité des fournisseurs. }\end{array}$ \\
\hline $\begin{array}{l}\text { 3. Rendement } \\
\text { Réduction des espaces } \\
\text { de production et } \\
\text { économies inhérentes. }\end{array}$ & $\begin{array}{l}\text { Coûts supplémentaires } \\
\text { de formation. }\end{array}$ & $\begin{array}{l}\text { Réduction ou élimination } \\
\text { des arrêts / retards } \\
\text { de production. }\end{array}$ & $\begin{array}{l}\text { Délais pour atteindre } \\
\text { le niveau optimal } \\
\text { de production. }\end{array}$ & & $\begin{array}{l}\text { Délais d'implantation } \\
\text { et d'adaptation. }\end{array}$ \\
\hline $\begin{array}{l}\text { 4. Délais de livraison } \\
\text { Rapprochement de l'en- } \\
\text { caissement des liquidités. }\end{array}$ & $\begin{array}{l}\text { Augmentation des frais } \\
\text { de livraison (pouvant être } \\
\text { pris en charge } \\
\text { par le donneur d'ordre). }\end{array}$ & $\begin{array}{l}\text { Augmentation } \\
\text { des ventes potentielles. }\end{array}$ & $\begin{array}{l}\text { Organisation du système } \\
\text { de livraison. }\end{array}$ & $\begin{array}{l}\text { Augmentation de la } \\
\text { satisfaction de la clientèle. }\end{array}$ & $\begin{array}{l}\text { Qualité des prévisions } \\
\text { initiales de la demande. }\end{array}$ \\
\hline 5. Flexibilité & $\begin{array}{l}\text { Coûts supplémentaires } \\
\text { imputables à la } \\
\text { convention collective. } \\
\text { Usure plus rapide des } \\
\text { équipements et machineries. }\end{array}$ & $\begin{array}{l}\text { Augmentation des ventes } \\
\text { par une production plus } \\
\text { flexible qui répond mieux } \\
\text { aux besoins des clients. }\end{array}$ & $\begin{array}{l}\text { Apprentissage du } \\
\text { fonctionnement des } \\
\text { nouveaux équipements. }\end{array}$ & $\begin{array}{l}\text { Intégration des fournisseurs } \\
\text { et des clients. } \\
\text { Polyvalence accrue } \\
\text { des employés et } \\
\text { des équipements. }\end{array}$ & $\begin{array}{l}\text { Réceptivité } \\
\text { des employés. } \\
\text { Non-implication des } \\
\text { dirigeants de l'entreprise. }\end{array}$ \\
\hline 6. Capacités innovatrices & $\begin{array}{l}\text { Coûts d'amélioration } \\
\text { de la conception } \\
\text { des produits et des } \\
\text { processus de production. }\end{array}$ & $\begin{array}{l}\text { Augmentation des ventes } \\
\text { par l'innovation apportée } \\
\text { aux produits fabriqués. }\end{array}$ & $\begin{array}{l}\text { Risque d'erreur } \\
\text { dans la conception. }\end{array}$ & $\begin{array}{l}\text { Augmentation du nombre } \\
\text { d'innovations possibles. }\end{array}$ & Image de l'entreprise. \\
\hline
\end{tabular}


voulant déterminer la rentabilité financière comme s'il s'agissait d'un projet industriel. Les quelques études conceptuelles relevées ne présentent pas une analyse complète de rentabilité qui tiendrait compte à la fois des coûts et des bénéfices quantifiables et non quantifiables (voir le tableau 2).

TABLEAU 2

Comparaison des différentes études

\begin{tabular}{|c|c|c|c|}
\hline & & $\begin{array}{l}\text { Analyse } \\
\text { descriptive }\end{array}$ & $\begin{array}{l}\text { Analyse } \\
\text { conceptuelle }\end{array}$ \\
\hline \multirow[t]{2}{*}{ Bénéfices } & Primaires & Corbey (1991) & $\begin{array}{l}\text { Jewkes et Power } \\
\text { (1993) }\end{array}$ \\
\hline & $\begin{array}{l}\text { Primaires } \\
\text { et secondaires }\end{array}$ & Wantuck (1989) & $\begin{array}{l}\text { Skank } \\
\text { et Govindarajan } \\
(1992)\end{array}$ \\
\hline \multirow[t]{2}{*}{ Coûts et Bénéfices } & Primaires & CMA (1993) & \\
\hline & $\begin{array}{l}\text { Primaires } \\
\text { et secondaires }\end{array}$ & Primrose (1992) & Accola (1994) \\
\hline
\end{tabular}

L'analyse d'un projet dans un environnement incertain requiert que l'on considère cette dimension dans l'évaluation de la rentabilité financière. Le tableau 1 peut permettre, jusqu'à un certain point, d'identifier les principaux facteurs de risque d'un projet d'implantation d'un système de gestion JAT. En fait, les bénéfices ainsi que les coûts qualitatifs peuvent être considérés comme des éléments de risque étant donné les difficultés d'estimation. Il en est de même pour les bénéfices et coûts secondaires que l'on ne réussit à estimer qu'avec un faible degré de précision. Plus l'entreprise aura à tenir compte d'éléments difficilement, voire pas du tout quantifiables, plus le projet sera risqué et devra être analysé soigneusement.

Nous proposons donc de montrer, à l'aide de cette grille et d'un exemple que nous avons construit à partir de nos entrevues, comment les méthodes traditionnelles de considération de la rentabilité d'un projet d'innovation de procédé peuvent amener à sous- ou surévaluer de façon notable les bénéfices actualisés attendus, et comment la prise en compte des éléments secondaires et qualitatifs peut nuancer la décision du dirigeant.

\subsection{Présentation des données et résultats des simulations}

Notre méthode d'évaluation propose donc de prendre en considération l'ensemble de ces éléments en quantifiant, à l'aide d'intervalles plus ou moins grands, les bénéfices et les coûts secondaires alors que les éléments qualitatifs seront considérés différemment. Nous voulons démontrer, par notre simulation, 
qu'il est erroné de ne considérer que les éléments primaires dans l'évaluation et que l'utilisation d'un taux d'actualisation ajusté ne permet pas de prendre en considération le risque total que représente un tel projet.

Nous avons réalisé des entrevues auprès de dirigeants d'entreprises manufacturières, liées entre elles par le fait qu'elles sont toutes des fournisseurs d'un même donneur d'ordre, qui avaient implanté ou étaient en voie d'implanter le JAT. Ces entreprises ont un taux de dépendance variant de 50 à $90 \%$ à l'égard de leur client principal ; elles comptent entre 75 et 200 employés et ont un chiffre d'affaires compris entre 5 et 10 millions de dollars. À partir de ces entrevues, des états financiers ainsi que des différents registres comptables de certaines des entreprises consultées et de la littérature en production, nous avons réussi à construire un scénario global réaliste susceptible de représenter correctement l'ensemble des bénéfices et des coûts primaires et secondaires qu'entraîne l'implantation d'un système de gestion juste-à-temps dans les PME manufacturières.

Dans le tableau 3, nous présentons les intervalles des montants utilisés pour la première et la dernière année du projet. Certains des montants indiqués croîtront pendant la durée du projet, alors que d'autres diminueront, et ce, conformément à ce qui nous a été révélé en entrevues et à ce que rapporte la littérature pertinente. C'est le cas, par exemple, des bénéfices attribuables à une augmentation du rendement, lesquels passeront graduellement de $30000 \$$ à $62000 \$$, alors que ceux imputables à la variation du coût de revient évolueront de $200000 \$$ vers $85000 \$$ en fin de projet. Par ailleurs, l'évolution des montants n'est pas nécessairement linéaire, puisque certains de ceux-ci connaîtront des fluctuations importantes dans les deux premières années de réalisation du projet, pour ensuite évoluer de façon plus régulière. Cette situation est applicable, entre autres, aux montants identifiés pour le coût de revient, lesquels passent (bénéfice maximal) de $200000 \$$ à 300000 à l'année 2, puis reviennent à $200000 \$$ avant de se stabiliser à $85000 \$$ pour les quatre dernières années. Ces données devraient refléter le rythme de progression attendu du projet.

Nous avons utilisé un horizon de sept ans pour déterminer la rentabilité globale du projet d'implantation. Jewkes et Power (1993) insistent sur le fait que l'on doit envisager, dans un tel projet, un calcul de rentabilité à long terme (horizon de 7 à 10 ans), puisque les principaux bénéfices attendus ne sont pas instantanés et prennent un certain temps avant de se matérialiser; les flux monétaires cumulés étant négatifs pour une certaine période. Rappelons l'exemple utilisé le plus souvent pour vanter les mérites du JAT : la réduction des temps de production à l'usine de Toyota, qui sont passés de 8 heures à 2 minutes. La plupart des tenants du JAT oublient toutefois de mentionner que cette réduction de temps a été obtenue sur une période de 15 ans et au prix 
d'importants investissements dans des moyens de production très flexibles !!! (voir Corbey, 1991).

Finalement, pour respecter le risque global d'une PME, nous avons choisi un taux d'actualisation de base de $20 \% 6$ auquel nous ajouterons, lorsque cela sera nécessaire, une prime de risque de $5 \%$.

TABLEAU 3

Éléments de bénéfices et de coûts utilisés dans la simulation

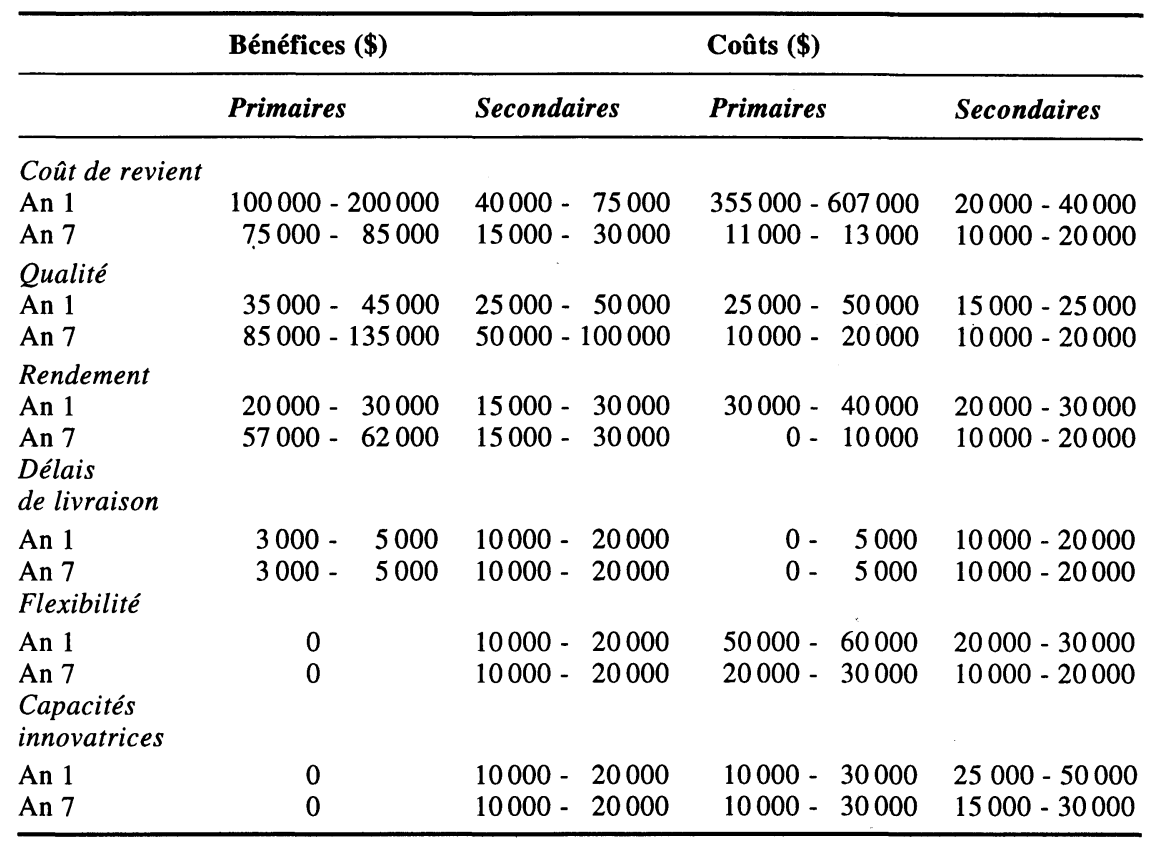

N.B. Les entrevues réalisées avec les dirigeants d'entreprises, ainsi que l'ensemble des éléments de la littérature consultée nous ont permis de quantifier les montants présentés ci-dessus. Notre démarche ne vise pas à évaluer correctement et avec précision la rentabilité financière d'un projet d'implantation d'un mode de gestion juste-à-temps, mais plutôt à souligner les erreurs méthodologiques si l'outil d'analyse ne convient pas. Nous n' accorderons donc qu'une attention mineure aux montants utilisés pour les fins de l'évaluation.

En utilisant ces informations, et la formule de la VAN présentée plus haut, on a pu obtenir différentes estimations pour le projet étudié. Nous avons utilisé les bénéfices maximaux possibles (fourchette supérieure de l'intervalle) et les coûts minimaux (fourchette inférieure) pour nous donner une VAN

6. Ce taux d'actualisation a pu être déterminé par nos entrevues avec des dirigeants de PME. Nous les avons interrogés individuellement sur le taux de rendement qu'ils exigent de leur entreprise. Unanimement, ils ont répondu : autour de $20 \%$. 
maximale. Le cas inverse a produit une VAN minimale. Finalement, tout comme le font certains auteurs, nous avons d'abord utilisé un taux d'actualisation $(20 \%)$ auquel nous avons ajouté une prime de risque $(5 \%)$ pour tenir compte des éléments difficilement quantifiables afin de produire une première évaluation du projet. Nous avons par la suite tenu compte des éléments primaires et secondaires que nous avons actualisés au taux de base $(20 \%)$. Cette deuxième série de calculs permet de tenir compte des commentaires d'Accola concernant la prise en compte du risque : ou bien on utilise un taux d'actualisation ajusté pour l'évaluation des flux monétaires primaires, ou bien on actualise au taux de base des flux monétaires plus conservateurs. Le tableau 4 présente les résultats obtenus.

TABLEAU 4

Différentes estimations pour la rentabilité du JAT

\begin{tabular}{|c|c|c|c|}
\hline & & VAN & $\begin{array}{l}\text { Délai de } \\
\text { récupération }\end{array}$ \\
\hline $\begin{array}{l}\text { Actualisation au taux de } 25 \% \\
\text { des bénéfices et des coûts primaires. }\end{array}$ & $\begin{array}{l}\text { Minimum } \\
\text { Maximum }\end{array}$ & $\begin{array}{c}(476844 \$) \\
220028 \$\end{array}$ & $\begin{array}{l}\text { plus de } 7 \text { ans } \\
\text { moins de } 3 \text { ans }\end{array}$ \\
\hline $\begin{array}{l}\text { Actualisation au taux de } 20 \% \\
\text { des bénéfices et des coûts } \\
\text { primaires et secondaires }\end{array}$ & $\begin{array}{l}\text { Minimum } \\
\text { Maximum }\end{array}$ & $\begin{array}{c}(609970 \$) \\
698689 \$\end{array}$ & $\begin{array}{l}\text { plus de } 7 \text { ans } \\
\text { moins de } 2 \text { ans }\end{array}$ \\
\hline
\end{tabular}

On voit que la considération des éléments secondaires, même en réduisant le taux d'actualisation, ne donne pas une estimation semblable à celle obtenue uniquement avec les éléments primaires, mais présente plutôt l'image d'un projet plus risqué, tout comme le suggéraient Primrose (1992) et Accola (1994). En effet, la distribution des VAN s'est étendue considérablement (écart entre les valeurs maximales et minimales), ce qui confirme les conclusions d'Accola sur l'importance de considérer de façon particulière les éléments difficilement quantifiables et que, justement parce qu'ils sont difficilement quantifiables, on ne réussit pas très bien à exprimer cette difficulté dans la prime de risque que l'on ajoute au taux de base. En fait, pour rendre équivalentes les deux solutions, il aurait fallu utiliser une prime de risque d'une ampleur de $35 \%$ (avec taux d'actualisation de 55,19\%) dans l'actualisation des bénéfices et coûts primaires et secondaires, ce que l'on ne retrouve jamais dans l'évaluation des projets même très risqués. Accola (1994) souligne également le fait que la prime de risque utilisée est déterminée bien plus par l'attitude des dirigeants envers le risque que par une représentation explicite du risque inhérent au projet. 
Une dimension non négligeable du JAT, surtout pour les entreprises qui en sont au tout début du processus d'innovation, a trait aux éléments qualitatifs qui, tel que nous l'avons précisé auparavant, contribuent à accroître le degré d'incertitude et donc de risque du projet. Nos entrevues ont permis de faire ressortir un certain pessimisme quant à la parfaite synchronisation des avantages et des coûts du JAT. De même, pour plusieurs des dirigeants rencontrés, la polyvalence et l'intégration des employés sont loin d'être assurées. Le phénomène était encore plus probant chez les entreprises syndiquées.

Nous n'avons pas essayé de quantifier cet aspect qualitatif, mais avons plutôt considéré son influence sur la structure temporelle des flux monétaires du projet. Nous avons donc reporté de 2 ans $^{7}$, comme le suggèrent Jewkes et Power (1993), le début de la matérialisation des bénéfices et, sans modifier les montants ayant servi à la simulation, avons recalculé la valeur actuelle nette (la période d'évaluation a été portée de 7 à 9 ans). En utilisant les valeurs maximales pour les bénéfices et minimales pour les coûts, nous obtenons une $\mathrm{VAN}_{\text {max. }}=225943 \$$. Par contre, le délai de récupération s'établit à près de 6 ans, comme le montre la figure 1, ce qui est trois fois plus long que le projet évalué de façon standard, qui présente un délai de 2 ans.

FIGURE 1

Valeur actuelle nette du JAT (prise en compte des éléments qualitatifs)

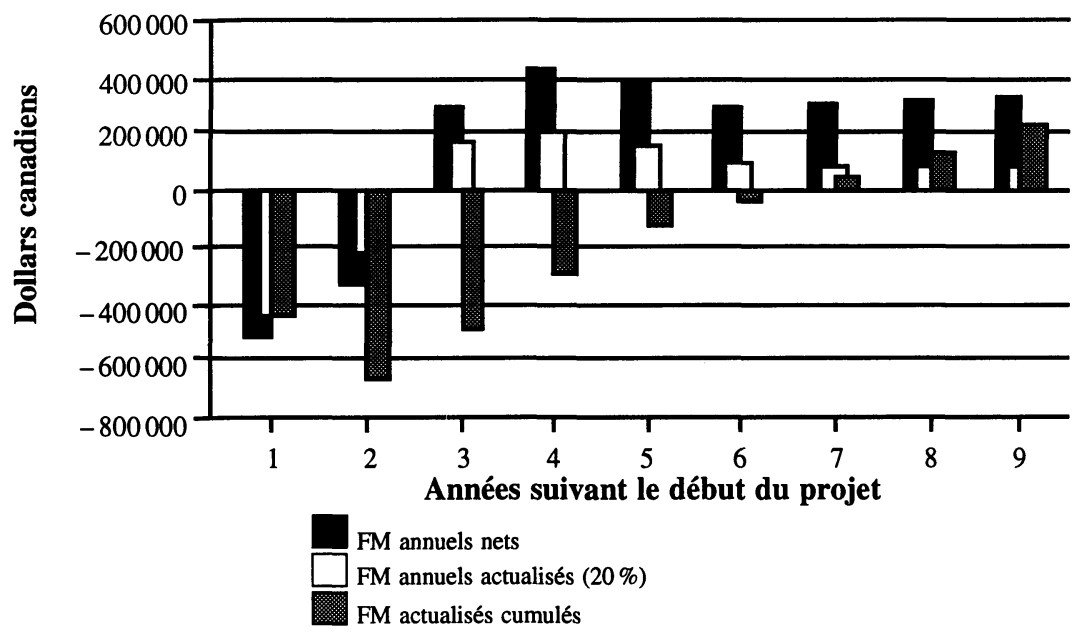

7. L'utilisation d'un délai supérieur à deux ans aurait rendu nos résultats encore plus probants. 
Le calcul du délai de récupération et la représentation temporelle des flux monétaires font clairement ressortir le niveau de risque d'un tel projet. Aucune étude n'a, jusqu'à présent, utilisé cette approche pour quantifier la rentabilité d'un projet d'innovation de procédé et ainsi fournir de meilleurs outils pour la prise de décision au dirigeant de la PME. Pis encore, aucune étude n'a apporté ces nuances lorsqu'on doit évaluer ce type de projet. On se souviendra, comme nous l'avons mentionné à la section 2, que l'une des principales causes d'échec des projets était attribuable au fait que les entrées de fonds tardaient à se réaliser. C'est donc cette situation que nous avons voulu évaluer en utilisant une approche de gestion de projets (Giard, 1991). Un traitement plus poussé des éléments non quantifiables aurait nécessité des efforts pour leur attribuer des valeurs numériques ${ }^{8}$, ce qui n'aurait probablement pas modifié les conclusions auxquelles nous sommes arrivés.

\section{Conclusion}

Les résultats de notre simulation font ressortir l'importance de l'évaluation globale et de la prise en compte de l'ensemble des bénéfices et des coûts facilement et difficilement quantifiables des projets d'innovation (que certains auteurs qualifient de projets immatériels), et que l'utilisation d'un taux d'actualisation arbitraire pour prendre en considération le risque est souvent insuffisante et peut mener l'évaluateur à des conclusions erronées. De même, nous avons indiqué l'importance d'introduire les éléments qualitatifs dans les résultats de l'évaluation afin de donner une image le plus fidèle possible, de l'ensemble du rendement et des éléments de risque du projet étudié.

Ces résultats confirment l'importance de procéder à l'analyse de la rentabilité financière de ce type de projet, comme s'il s'agissait d'un projet conventionnel tel que l'agrandissement d'une usine ou l'achat de nouveaux équipements de production.

La difficulté à quantifier certains éléments d'un projet ne justifie pas l'absence d'une analyse de rentabilité financière. Bien que les modèles traditionnels de choix d'investissement ne soient pas adaptés aux projets d'innovation, on peut utiliser un modèle plus complet incluant l'ensemble des éléments

8. Certains logiciels de gestion de projets (notamment de traitement du risque dans les projets) permettent de définir des distributions de probabilités pour les variables que l'on ne peut quantifier avec un très grand degré de précision. On peut ensuite réaliser des simulations sur les résultats obtenus, et identifier les variables critiques du projet. Cette procédure permet de fournir aux décideurs un éventail de scénarios possibles, selon différents états de la nature qu'ils croient susceptibles de se réaliser. N'oublions pas que l'analyse financière ne vise qu'à mieux éclairer le dirigeant dans sa prise de décision, et que cette dernière lui appartient. 
primaires, secondaires et qualitatifs afin de mesurer le rendement espéré et le risque associé à ce type d'investissement. Accepter de ne pas procéder à une bonne analyse de rentabilité financière, c'est prendre le risque de ne pas optimiser l'allocation des ressources à l'intérieur de l'entreprise.

Cela est d'autant plus vrai que les quelques études réalisées jusqu'à présent sur les effets du juste-à-temps sur la rentabilité des entreprises sont loin d'être convaincantes.

Balakrishnan et al. (1994) ont mesuré si les entreprises ayant adopté le JAT avaient connu une meilleure rentabilité. Selon les résultats obtenus pour l'ensemble de l'échantillon, l'implantation du JAT n'a pas eu d'influence sur la rentabilité. Par contre, lorsqu'ils tiennent compte de la relation de dépendance entre les fournisseurs et un donneur d'ordre important et qu'ils séparent leur échantillon entre des entreprises dépendantes d'un donneur d'ordre et d'autres indépendantes, les résultats indiquent que celles qui sont dépendantes ont connu une diminution de leur profitabilité plus forte que celles qui sont indépendantes. Ces résultats autorisent à penser que certaines caractéristiques ignorées jusqu'à présent, telles que le type de produit, le marché, la concentration de la clientèle, sont autant de facteurs qui peuvent influencer de façon notable les profits que peut engendrer le JAT.

L'étude de Inman et Mehra (1993) permet de nuancer et de réduire l'ampleur des avantages présumés du JAT. Toutefois, d'autres études dans la même direction devraient prendre en considération des variables propres à la firme et à son environnement afin de voir si les mêmes bénéfices peuvent être réalisés par l'ensemble des entreprises.

Étant donné la croissance des activités de recherche et de développement dans les PME ainsi que le nombre d'innovations de procédés et de produits auxquelles elles participent, les recherches en évaluation financière doivent se poursuivre afin de raffiner les méthodes d'analyse de rentabilité et surtout de la prise en compte du risque. Le rôle des chercheurs est de développer des outils d'analyse efficaces et de convaincre les entrepreneurs de l'importance de l'analyse de faisabilité financière. Un projet d'innovation, bien que difficilement quantifiable dans son ensemble, mérite une évaluation préalable, sinon il risque plus de freiner l'entreprise que de la faire progresser.

\section{Bibliographie}

ACCOLA, W.L. (1994), «Assessing risk and uncertainty in new technology investments», Accounting Horizons, vol. 8, $\mathrm{n}^{\circ} 3$, p. 19-35.

BALAKRISHNAN, R., T.J. Linsmeier et M. VenkATAChAMAM (1994), "Sharing financial benefits from improved inventory utilisation : effect of product-market characteristics », Document de travail, The University of Iowa, 35 pages. 
Behrens, W. et P.M. HaWranex (1993), Manuel de préparation des études de faisabilité industrielle, Organisation des Nations Unies pour le développement industriel, Vienne, 394 pages.

BILLESBACH, T.J. et R. HAYEN (1994), «Long-Term impact of just-in-time on inventory performance measures ", Production and Inventory Management Journal, vol. 1, p. 62-67.

CMA (1993), Politique de comptabilité de management: La mise en application des systèmes de production juste-à-temps, Société des comptables en management du Canada, 54 pages.

CORBEY, M. (1991), « Measurable economic consequences of investments in flexible capacity », International Journal of Production Economics, vol. 23, p. 47-57.

GÉlinas, R., R. JaCoB, J. Drolet et M. Rheault (1994), «Les facteurs de succès du juste-à-temps et leur incidence sur les PME», Cahier de recherche no 94-01, GREPME, Université du Québec à Trois-Rivières, Québec, 21 pages.

GIARD, V. (1991), Gestion de projets, Paris, Economica, 174 pages.

INMAN, R.A. et S. MEHRA (1993), «Financial justification of JIT implementation », International Journal of Operations and Production Management, vol. 13, $\mathrm{n}^{\circ} 4$, p. 32-39.

JEWKES, E. et M. POWER (1993), «A microeconomic analysis of investment in just-in-time manufacturing », International Journal of Production Economics, vol. 29 , p. 313-321.

JoG, V.M. et A.K. SRIVASTAVA (1994), «Corporate financial decision making in Canada », Canadian Journal of Administrative Sciences, vol. 11, n 2, p. 156-176.

MACKEY, J.T. (1989), «JIT jitters about responsibility accounting », CA Magazine, p. 22-25.

MiLtenbURG, J. (1993), «A theoretical framework for understanding why JIT reduces cost and cycle time and improves quality ", International Journal of Production Economics, vol. 30-31, p. 195-204.

Philipoom, P.R., L.P. ReES, B.W. TAYlor et P.Y. HuANG (1987), « An investigation of the factors influencing the number of Kanbans required in the implementation of the JIT technique with Kanbans », International Journal of Production Research, vol. 25, $\mathrm{n}^{\circ} 3$, p. 457-472.

Pinsonnault, F. (1996), «Appréciation financière des projets de conversion vers une production à valeur ajoutée en PME manufacturières », Rapport de recherche, Programme de maîtrise en gestion de projet, Université du Québec à Trois-Rivières, Québec, 200 pages.

PRIMrose, P.L. (1992), «Evaluating the introduction of JIT», International Journal of Production Economics, vol. 27, p. 9-22. 
RAMUdhin, A. et P. MARIER (1992), «JITSIM : un progiciel orienté-objet pour la simulation du juste-à-temps dans l'industrie manufacturière », Document de travail 92-31, Université Laval, Québec, 11 pages.

SHANK, J.K. et V. GoVINDARAJAN (1992), « Strategic cost analysis of technological investments », Sloan Management Review, 39-51.

ST-PIERRE, J. et R. BEAUdOIN (1995), « La prise en compte du risque dans un projet d'innovation de procédé dans les PME manufacturières », Actes du colloque $d u$ deuxième congrès international francophone de la PME, Paris, p. 256-276.

Wantuck, K.A. (1989), Just-In-Time for America: A Common Sense Production Strategy, KWA Media, Michigan, 423 pages. 